\title{
Situación actual de la Educación Médica en Chile
}

\author{
Comité de Educación Superior, de la Academia Chilena de \\ Medicina
}

\section{Current Status of Medical Education in Chile}

The Chilean Academy of Medicine published a "Report on the Current Status of Medical Education in Chile". This report reviews the history of medical education in this country and its close relationship with the Health Care System, public and private; highlights the main changes that took place during the last 25 years in superior and medical education; provides information on the 26 currently existing Medical Schools; refers to the availability of medical doctors and specialists; discusses the mechanisms that control the quality of institutions involved and their programs; and summarizes the results of the Annual National Medical Examination. The members of the Committee on Superior Education of the Academy provided a critical analysis of medical education in Chile and recommendations on how to improve it. Revista Médica de Chile reproduces the Summary, Conclusions and Recommendations. The complete document (in Spanish) can be accessed freely at www.institutodechile.cl (Rev Méd Chile 2009; 137: 709-12).

(Key words: Delivery of Health Care; Education, medical, undergraduate; Internship and Residency).

L a Academia Chilena de Medicina ha publicado ـun "Informe sobre la situación actual de la educación médica en Chile". El informe da cuenta de los antecedentes históricos de la educación médica y su relación con el sector salud; los principales cambios que han ocurrido en los últimos 25 años en educación superior y médica; entrega numerosos antecedentes sobre las 26 escuelas de medicina existentes en el país; la disponibilidad de médicos y especialistas; los mecanismos de control de calidad de instituciones y programas y los resultados del examen médico nacional. Se hace un análisis de la educación médica y se formulan recomendaciones para

Correspondencia a: Dr. Rodolfo Armas M. Academia Chilena de Medicina. Almirante Montt 453, Santiago de Chile.

E mail: cblamey@tie.cl

El documento "Informe sobre la Situación Actual de la Educación Médica en Chile" fue elaborado por el Comité de Educación Superior de la Academia Chilena de Medicina, integrado por los Académicos Dres. Rodolfo Armas M. (Presidente del Comité), Alejandro Goic G., Colomba Norero V., José Adolfo Rodríguez P., Eduardo Rosselot J., Sylvia Segovia P. (Q.E.P.D.) y Vicente Valdivieso D. El texto completo fue publicado el año 2008 en la Serie Monografías Académicas, de la Academia de Medicina del Instituto de Chile y tiene acceso libre en la página web www.institutodechile.cl 
mejorarla. La publicación incluye una extensa bibliografía sobre la materia. A continuación presentamos el resumen ejecutivo de este documento.

\section{RESUMEN EJECUTIVO}

La educación médica en el país tiene antecedentes remotos en la Universidad de San Felipe (1756), pero se inició formalmente con el Curso de Medicina de 1833 en el Instituto Nacional, que fue traspasado luego a la Universidad de Chile (1842). En las primeras décadas del siglo XX se crearon las Facultades de Medicina de Concepción (1924), de la Pontificia Universidad Católica de Chile, en Santiago (1929) y, posteriormente, las de las Universidades Austral de Chile, en Valdivia (1967), de Valparaíso, en esa ciudad, y de La Frontera, en Temuco (1981). La relación entre estas escuelas y el sistema sanitario público y la selectividad del ingreso de alumnos a los estudios de medicina, fueron caracteństicas muy relevantes de los primeros 150 años de docencia médica. De hecho, ya en 1930 la Escuela de Medicina de la Universidad de Chile implantó la selección estricta de un número limitado de alumnos.

A partir de la década de 1980, aumentó el número de Escuelas de Medicina, predominantemente privadas $\mathrm{y}$, consecuentemente el número de estudiantes. Actualmente existen 26 Escuelas pertenecientes a 20 universidades que, en conjunto, tienen una matrícula de 1.682 alumnos y un total de 10.000 alumnos. Las Escuelas difieren en los puntajes de ingreso de sus alumnos (Prueba de Selección Universitaria, PSU), los costos de la educación, la disponibilidad de campos clínicos, los recursos docentes y las actividades de investigación. Las Escuelas más tradicionales son las que tienen mayor número de alumnos, muestran más docentes de jornada completa o media jornada, mayor disponibilidad de campos clínicos y son las únicas que realizan investigación significativa.

En los últimos 17 años se han ido implementando sistemas rigurosos de acreditación de las Universidades y de las Escuelas de Medicina y se ha instituido un Examen Médico Nacional que rinden todos los egresados y cuyos resultados de los últimos cinco años se muestran en el documento.

Actualmente Chile dispone de 25.542 médicos, siendo 9.627 de ellos especialistas certificados; la relación médico-habitantes ha mejorado sosteni- damente, llegando a un profesional por cada 650 habitantes. El número de médicos extranjeros avecindados en Chile ha aumentado, los que se desempeñan especialmente en el sector público en la atención primaria. La mayoría de ellos no han sido evaluados por acogerse a antiguos tratados internacionales que los favorecen.

En el último cuarto de siglo, el número de universidades ha aumentado de 8 a 62, las Escuelas de Medicina de 8 a 26 y la población de jóvenes que siguen estudios superiores ha crecido más de cinco veces. En las universidades públicas, los estudiantes deben asumir el costo de los estudios y un sistema de becas y créditos ayuda a los de menores recursos económicos. La mayor cobertura de la educación superior en el país y la descentralización de instituciones han contribuido al progreso cultural y técnico de las regiones. La medicina no ha sido ajena a esos progresos, pero han surgido situaciones complejas respecto a los campos clínicos y a las relaciones entre las Escuelas de Medicina y las autoridades locales de los Servicios de Salud.

A juicio de la Academia, es indispensable implementar una serie de medidas para el perfeccionamiento de la educación médica en el país. Ellas son: establecer mecanismos que proporcionen información actualizada, confiable y oportuna sobre el número de médicos y especialistas y su distribución geográfica; reforzar los sistemas de acreditación, con participación de las principales instituciones médicas nacionales y difundir públicamente sus resultados; someter los proyectos de nuevas Escuelas de Medicina a un proceso de supervisión continua hasta el logro de su autonomía (Licenciamiento); cerrar las Escuelas de Medicina que no logren la acreditación; reactivar la Comisión Nacional Docente Asistencial en Salud (CONDAS) y las comisiones locales coordinadoras entre las Escuelas y los Servicios de Salud; incentivar a los médicos chilenos para que ejerzan la atención primaria, a través de estímulos profesionales, técnicos y económicos, y valorar esta actividad en los programas de pregrado; rehabilitar física y tecnológicamente los hospitales públicos en los que se realiza docencia; exigir la dedicación exclusiva en los programas de formación de médicos especialistas (Residencias) y reestudiar su financiamiento; revisar los tratados internacionales sobre reconocimiento del título de médico-cirujano de los graduados en el extranjero y -dado el alto costo 
que han alcanzado las matrículas y arancelesgarantizar por parte de la sociedad que quienes sean académicamente capacitados puedan estudiar aunque carezcan de recursos económicos.

El informe incluye una extensa bibliografía nacional sobre educación médica, mucha de la cual corresponde a publicaciones y estudios elaborados a través de los años por miembros de la Academia de Medicina.

\section{CONCLUSIONES Y RECOMENDACIONES}

1. Chile está viviendo profundos cambios en todo el espectro de la educación superior, incluida la enseñanza de la medicina, los que no son exclusivos de nuestro país. Los médicos y sus instituciones tienen la responsabilidad de velar por la calidad de la educación médica y la obligación moral de responder a la fe pública que en ellos deposita la sociedad chilena.

El conjunto de modificaciones en el sistema de educación superior que se ha producido en los últimos 25 años, ha tenido algunos aspectos muy beneficiosos como son la mayor cobertura educacional, que ha dado acceso a un número creciente y significativo de los jóvenes a la educación superior; la instalación de Universidades y de Escuelas de Medicina en lugares donde ellas no existían, contribuyendo al mejoramiento cultural y técnico de las diversas regiones; la incorporación de recursos privados en la educación superior y el mejoramiento de la relación en el número de médicos por habitantes. La tarea prioritaria de hoy es velar por la calidad de la educación médica que se imparte.

2. Se carece de información confiable, actualizada y oportuna acerca de la cantidad y distribución geográfica de médicos y especialistas en Chile. Es indispensable y urgente que las autoridades de salud implementen un sistema para contar con esta información, así como con la proyección de los recursos profesionales en el mediano plazo. Ello permitińa enfrentar oportunamente el déficit actual según especialidades y regiones y adecuar los cupos en los programas de formación de especialistas a las necesidades reales del país.

3. El costo de los estudios de medicina para los alumnos y sus familias en términos del valor de la matrícula y los aranceles ha tenido un significativo incremento en los últimos años, lo que implica el riesgo de que estudiantes idóneos no puedan ingresar a las Escuelas de Medicina por razones económicas.

Es muy deseable que la sociedad garantice que las limitaciones de orden económico no excluyan de los estudios médicos a alumnos académicamente capaces y con vocación de servicio. Un sistema de becas o de créditos con pago diferido de matrículas y aranceles para cuando los alumnos se incorporen al mundo del trabajo, son mecanismos que adecuadamente implementados pueden garantizar que los mejores estudiantes ingresen a las Escuelas de Medicina. Las Escuelas de Medicina tradicionales, y muy particularmente las de carácter público, tienen la obligación de constituirse en un mecanismo moderador del costo de los estudios médicos.

4. No parece razonable que la atención primaria, que tiene alta prioridad en la atención de salud de la población, esté en una proporción significativa en manos de médicos extranjeros que, mayoritariamente, han ingresado al ejercicio profesional sin ser evaluados. También es inquietante que los médicos chilenos demuestren escaso interés en participar en la atención primaria.

En correspondencia con lo anterior las Facultades de Medicina debieran evaluar si están impartiendo una docencia que capacite adecuadamente y haga atractiva para los estudiantes la atención primaria, y las autoridades sanitarias preguntarse si esa es una actividad asistencial que cuenta con los incentivos profesionales y económicos correctos.

5. No es sensato que médicos graduados en el extranjero puedan ejercer la profesión en Chile sin que hayan sido sometidos a una evaluación de sus competencias y con el mero registro del título en el Ministerio de Relaciones Exteriores. La Academia de Medicina estima indispensable que se revisen aquellos tratados internacionales que permiten el ejercicio profesional en Chile a médicos poseedores del título de médico-cirujano, obtenido en algunos países extranjeros. Estos convenios recíprocos son de antigua data y no resultan equitativos respecto de las exigencias académicas a que se someten los médicos chilenos y los extranjeros que 
revalidan su título en la Universidad de Chile, previa evaluación de sus competencias.

6. La relación que desde hace décadas ha existido entre las Facultades de Medicina y el sector público de salud ha sido extraordinariamente beneficiosa para el país. Muchos de los logros en salud y educación médica no habrían sido posibles sin ella, entre otros la mejoría progresiva y significativa en la relación médicohabitantes. Lamentablemente esta relación se ha deteriorado en el transcurso de los años.

Es necesario que la autoridad de Salud reactive $\mathrm{y}$ asegure el funcionamiento permanente de las comisiones docentes asistenciales nacional y locales. La Comisión Nacional Docente Asistencial (CONDAS) integrada por autoridades del Ministerio de Salud, de las Facultades de Medicina y gremiales, podría resolver importantes materias de interés común para el sistema universitario y el sector estatal de salud, como el uso de los campos clínicos que pertenecen al Ministerio de Salud, el desinterés de los médicos chilenos por desempeñarse en la atención primaria, el estímulo a las investigaciones que tienen especial interés para mejorar la salud de la población y los requerimientos de médicos y especialistas.

Ambas instituciones debieran definir relacionarse a través de un convenio de largo plazo e informarse mutuamente de los planes y programas y de todas las materias de interés común y garantizar las facilidades básicas para los estudiantes como vestuarios, servicios higiénicos y alimentación cuando corresponda. Por su parte, la institución docente debe retribuir al centro asistencial por el beneficio que representa contar con un hospital 0 consultorio para la enseñanza de sus estudiantes e incentivar la participación de los médicos del centro asistencial en las actividades docentes, implementando estímulos económicos, académicos y profesionales.

7. Varios de los hospitales y consultorios públicos en que se realiza la docencia universitaria no reúnen las condiciones de infraestructura, implementación tecnológica, organización y funcionamiento para la adecuada enseñanza de los estudiantes de medicina y de los especialistas médicos.

Los futuros médicos debieran formarse en establecimientos docente-asistenciales que sean mo- delos de calidad de la atención médica, que cuenten con todas las disciplinas correspondientes a su nivel de complejidad y estén acreditados por un comité externo e independiente. Urge que la autoridad pública rehabilite los hospitales deteriorados y construya hospitales y consultorios modernos donde se requieren, dotados de tecnología de uso clínico actualizado, tanto para la debida atención de los enfermos como para la enseñanza de pre y postítulo.

8. Las principales instituciones médicas chilenas -Academia de Medicina, Facultades de Medicina, Colegio Médico de Chile y Sociedades Médicas- tienen el deber de seguir contribuyendo decisivamente a los controles de calidad de la educación médica en el país.

Los diversos sistemas de supervisión y control de calidad de la enseñanza médica que está en vigencia 0 en proceso de implementación deben mantenerse y perfeccionarse y no ceder en su rigurosidad. Las disposiciones de la Ley de Aseguramiento de la Calidad de la Educación Superior que establecen la acreditación obligatoria de las Escuelas de Medicina es un paso determinante en resguardo de la calidad de la educación médica.

Es deseable que los proyectos de nuevas escuelas de medicina sean sometidos a licenciamiento para garantizar que cuenten con los recursos necesarios para proporcionar una educación médica de calidad. Igualmente las escuelas que no logren acreditarse deberían ser excluidas del sistema educacional. Los servicios públicos de salud y las instituciones privadas asociadas a las escuelas de medicina, también debieran acreditarse por los organismos y sistemas que corresponda.

9. La formación de especialistas es un proceso que requiere dedicación de jornada completa y dedicación exclusiva para el adecuado cumplimiento de sus obligaciones académicas y asistenciales, teóricas y prácticas.

No es aceptable que en esta etapa de su formación los médicos tengan que distraer su tiempo para financiar su sustento y el pago de matrículas y aranceles, en actividades ajenas a su programa de especialización. Urge recuperar la dedicación exclusiva de los médicos en etapa de especialización que contemplaban originalmente estos programas. 